\title{
Improving the Performance of Facial Expression Recognition Using Dynamic, Subtle and Regional Features
}

\author{
Ligang Zhang and Dian Tjondronegoro \\ Queensland University of Technology, Brisbane, 4000, Australia \\ ligzhang@gmail.com,dian@qut.edu.au
}

\begin{abstract}
Human facial expression is a complex process characterized of dynamic, subtle and regional emotional features. State-of-the-art approaches on facial expression recognition (FER) have not fully utilized this kind of features to improve the recognition performance. This paper proposes an approach to overcome this limitation using patch-based 'salient' Gabor features. A set of 3D patches are extracted to represent the subtle and regional features, and then inputted into patch matching operations for capturing the dynamic features. Experimental results show a significant performance improvement of the proposed approach due to the use of the dynamic features. Performance comparison with pervious work also confirms that the proposed approach achieves the highest CRR reported to date on the JAFFE database and a toplevel performance on the Cohn-Kanade (CK) database.
\end{abstract}

Keywords: Facial expression recognition, Adaboost, support vector machine.

\section{Introduction}

Facial expression recognition (FER) is becoming an increasingly active research field in recent years due to its potential to be applied in many areas. FER supports many practical applications, such as human-computer interaction, patient and driver state detection. However, robust FER is still a challenging task as facial expression is a complex process, which is characteristic of different dynamic, subtle and regional facial changes (e.g. wrinkles) and is easy to be influenced by various environmental changes (e.g. illumination and occlusions). Therefore, an important step for FER is to accurately extract the useful dynamic, subtle and regional emotional features.

Current FER approaches can be classified into 4 categories based on the used feature types: motion-based, feature-based, model-based and appearance-based. Appearance-based approach can analyze facial images using multi-resolution information and has shown a significant advantage over other approaches in terms of capturing subtle features. The features used include Gabor feature [1], local binary patterns (LBP) [2], Haar [3], discrete Fourier transform (DFT) [4] etc. However, Current appearance-based approaches suffer from the drawback of using point-based Gabor or DFT features, which lack the ability to capture regional features. At the 
same time, the LBP and Haar are essentially based on statistics and cannot capture the subtle features with pixel accuracy. In addition, appearance-based approaches in static images have yet to consider the dynamic information of feature position, scale and shape changes, which also represent useful information for FER.

This paper proposes an FER approach to improve the recognition performance based on patch-based 'salient' Gabor features. The novelty of our approach lies in the adoption of patch-based Gabor features and the definition of patch matching to solve point-based Gabor features' limitation in capturing the dynamic, subtle and regional features. The experimental results demonstrate big performance improvements as well as the state-of-the-art performances of the proposed approach on both the JAFFE and the Cohn-Kanade (CK) databases. The rest of the paper is organized as follows. Section 2 presents the proposed approach. Section 3 gives the experimental results. The conclusions are drawn in Section 4.

\section{Proposed Approach}

Fig. 1 illustrates the proposed approach, which is composed of the pre-processing, training and test stages. At the pre-processing stage, facial regions are manually cropped to imitate rough face detection and scaled to a resolution of $48 * 48$ pixels. Then multi-resolution Gabor images are attained by convolving Gabor filters with these scaled facial regions. 2D Gabor filter with 8-scale (5:2:19 pixels) and 4orientation $\left(-45^{\circ}, 90^{\circ}, 45^{\circ}, 0^{\circ}\right)$ is used, and the other parameters are set based on [5]. During the training stage, a set of $3 \mathrm{D}$ patches are extracted from the Gabor images to represent the subtle and regional features. Patch matching operations are then performed to convert all extracted patches to distances for capturing the dynamic features of position, scale and shape changes. At the test stage, the distance features in a new image are attained by performing the same patch matching operations, and fed into support vector machine (SVM) to recognize 6 emotions: anger (AN), disgust (DI), fear (FE), happiness (HA), sadness (SA) and surprise (SU). The use of Gabor filter and SVM is due to their excellent performance reported in previous work [1]. Two SVMs with linear and radial basis function (RBF) kernels are used.

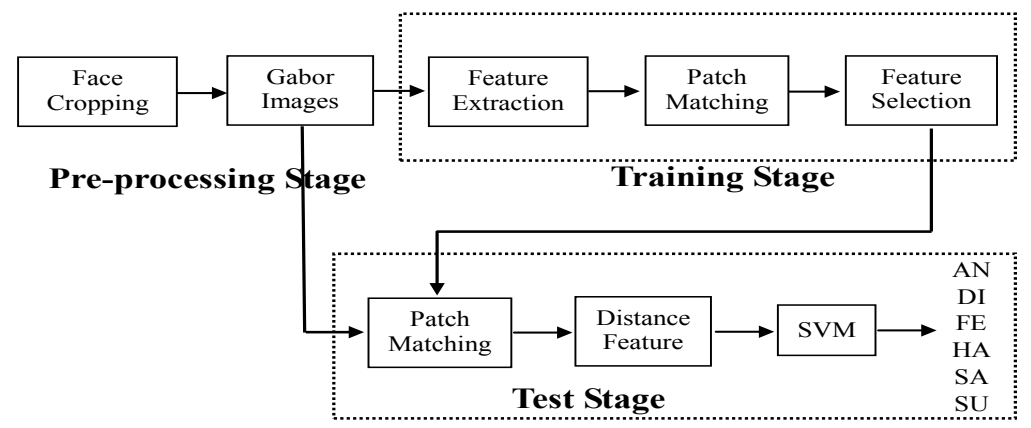

Fig. 1. Framework of the proposed approach. 


\subsection{Patch-based Feature Extraction}

Feature extraction generates a set of discriminating 3D patches, which have an advantage over point-based Gabor features to represent the subtle and regional emotional features. As shown in Fig. 2, the algorithm can be described as follows: (1) all training images are classified into 10 sets. For each emotion, each Gabor scale, and each patch size, one Gabor image is randomly selected from all images of the given emotion $E_{k}$. (2) Given one patch with the size of $P_{j}^{*} P_{j}^{*} O_{\text {num }}$ move this patch across the row and column pixels of this Gabor image, a set of patches can be extracted (the line $a$ ). (3) Record the corresponding matching area and matching scale (the line $b$ and $c$, details are explained in Section 2.2). (4) The final patch set can be constituted by concatenating the extracted patches of all emotions, all scales and all patch sizes.

To reduce the feature dimension and increase the processing speed, we only extract part of all patches by moving the patch $P_{a}$ with a step (i.e. Move_step in Fig.2). The patch sizes (width*height*orientation) and the corresponding moving steps are set to be $2 * 2 * 4,4 * 4 * 4,6 * 6 * 4,8 * 8 * 4$, and to be $1,2,3,4$ pixels respectively. Given $48 * 48$ images and 8-scale, 4-orientation Gabor filters, the final set contains 148,032 patches.

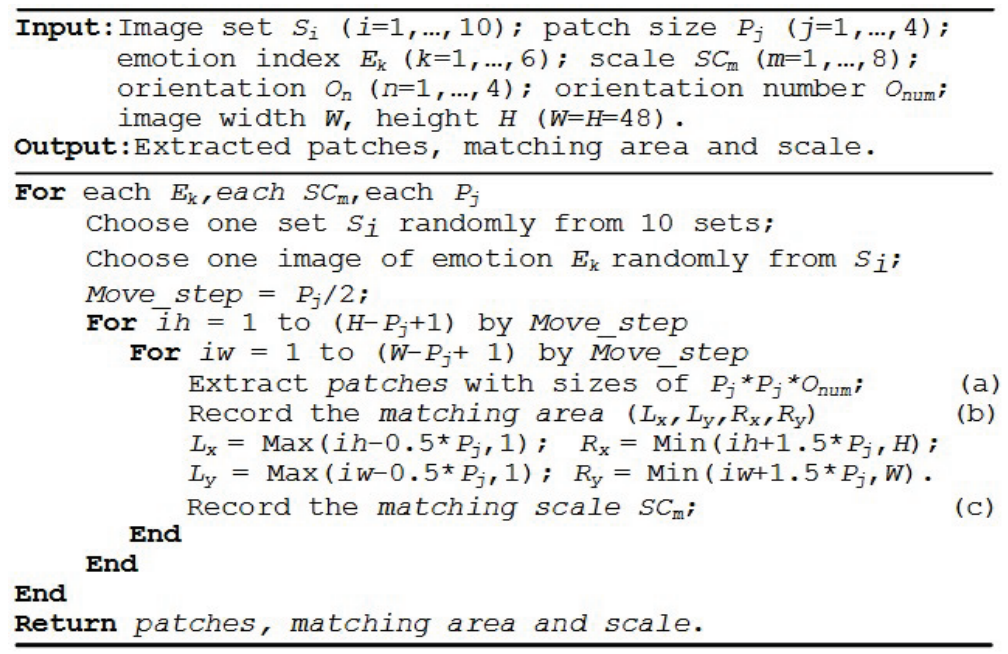

Fig. 2. Pseudo code of patch-based feature extraction.

\subsection{Patch Matching Operation}

Given the patch set obtained in feature extraction, the patch matching operation tends to convert it into distance features, which can capture the dynamic information of feature position, scale and shape changes. As shown in Fig. 3, the patch matching operation comprises of 4 steps for each patch and each training image: (1) matching area and matching scale are defined to provide a bigger matching space (Fig. 3 (c)). Based on this space, the emotional feature, which varies its position, scale and shape in different images, still can be captured provided that it is located within the space. (2) The distances are obtained by matching this patch with all patches within its matching 
space in the training image. This step takes two patches as inputs and yields one distance value based on a distance metric (Fig. 3 (b,d,e)). (3) The minimum distance is chosen as the distance feature for this patch and this training image (the black block in Fig. 3 (f)). (4) The distance features of all patches are combined into the final set with 148,032 elements (Fig. 3 (f)).

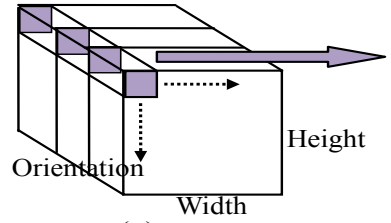

(a)

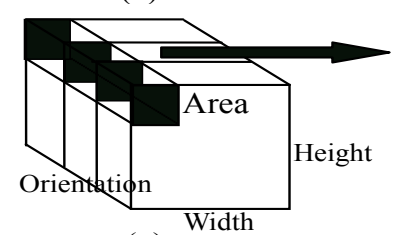

(c)

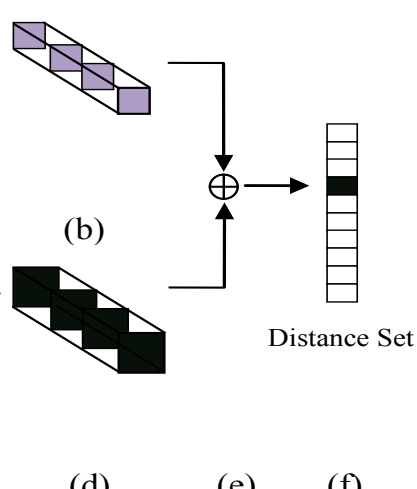

(d)

(e)

Fig. 3. Patch matching operation. One patch (b) is extracted from Gabor images (a); the corresponding matching area 'Area'(c); one distance (black block in (f)) is obtained by matching two patches (e).

The definition of matching area and matching scale plays a key role in capturing the dynamic features of position, scale and shape changes. The idea of them stems from the observation that position and scale of one feature do not have big changes in different facial images once these images are roughly located by a face detector. Thus, the invariance to position and scale changes can be accomplished by defining one such matching space for each feature. In this paper, given a patch $P_{a}$ with size $P_{j}{ }^{*} P_{j}{ }^{*} O_{\text {num }}$ its matching area is set 2 times of $P_{a}$ in width and height, but with the same orientation number and centre point. That is Area $=\left(2 * P_{j}\right) *\left(2 * P_{j}\right) * O_{\text {num }}$ While the matching scale is the same scale as patch $P_{a}$ because most cropped facial regions belong to the same scale. There are 4 distance metrics used: dense $\mathrm{L}_{1}\left(\mathrm{DL}_{1}\right)$, dense $\mathrm{L}_{2}$ $\left(\mathrm{DL}_{2}\right)$, sparse $\mathrm{L}_{1}\left(\mathrm{SL}_{1}\right)$ and sparse $\mathrm{L}_{2}\left(\mathrm{SL}_{2}\right)$. Sparse distance uses the maximum value of all orientations, while dense distance uses all values of all orientations.

\subsection{Salient' Patch Selection}

The feature extraction step produces a patch set that contains a big number of features and redundant information; therefore, this paper adopts the widely used and efficiency proved Adaboost for discriminative (called 'salient' here) patch selection. Since Adaboost was designed to solve two-class problems, in this research, the one-againstrest strategy is used to solve the six-emotion-class problem. The training process stops when the empirical error is below 0.0001 with an initial error of 1 . This setting is inspired by the stopping condition in [1] that the generalization error becomes flat. Regarding the training set, the JAFFE database includes all selected images, whereas the CK database is only composed of the peak frames. 
To explore the relationship between the CRR and the number of features, a group of error thresholds as listed in Table 1 are used to control the number of 'salient' patches. These thresholds are set based on our experimental observation that the empirical errors of Adaboost decrease with a factor of 10 and its numbers are evenly distributed between decimal intervals (e.g. 0.01 to 0.02 ). Accordingly, 38 numbers of features with 38 CRRs can be obtained by selecting patches with empirical errors bigger than the corresponding error thresholds. Note that " 0 " accepts all features.

Table 1. The 38 error thresholds used to control the number of patches.

\begin{tabular}{cc}
\hline Index & Error thresholds \\
\hline $1^{\text {st }}-10^{\text {th }}$ & $(10,9,8,7,6,5,4,3,2,1)^{*} 0.1$ \\
$11^{\text {th }}-19^{\text {th }}$ & $(9,8,7,6,5,4,3,2,1)^{*} 0.01$ \\
$20^{\text {th }}-28^{\text {th }}$ & $(9,8,7,6,5,4,3,2,1)^{*} 0.001$ \\
$29^{\text {th }}-38^{\text {th }}$ & $(9,8,7,6,5,4,3,2,1,0)^{*} 0.0001$ \\
\hline
\end{tabular}

\section{Experimental Results}

\subsection{Databases}

The JAFFE database [6] contains 213 gray images of 7 expressions posed by 10 Japanese females. Each object has 3 or 4 frontal face images for each expression and their faces are approximately located in the middle of the images. All images have been rated on 6 emotion adjectives by 60 subjects. The released portion of the CohnKanade (CK) database [7] includes 2105 digitized image sequences from 182 subjects ranged in age from 18 to 30 years. The 6 basic expressions were based on descriptions of prototypic emotions. Image sequences are shown from neutral to target emotion.

In this paper, all the images of 6 expressions from JAFFE are used. For CK, 1,184 images that represent one of the 6 expressions are selected, 4 images for each expression of totally 92 subjects. The images are chosen from the last image (peak) of each sequence, then one every two images. The face regions of all selected images are cropped by taking the nose as a center point, and scaled to $48 * 48$ pixels. The resulting regions contain features with different positions, scales and shapes.

\subsection{Performance on the JAFFE Database}

Fig. 4 shows the relationship between the CRR and the number of features on the JAFFE database. The CRR is the average performance of 10 -set cross-validation. As can be seen, the proposed approach achieves the highest CRR of $93.48 \%$ using $\mathrm{DL}_{2}$ and linear SVM when the error threshold equals to 0.0001 and the number of features equals to 185 . The overall performances of 4 distances grow up rapidly at the starting stage, however, the performances begin to level off when the number of features exceeds 150 for linear and 80 for RBF. For the performances of SVMs, linear performs better than RBF for all distances. Regarding the overall performances of distances, for both linear and RBF, the best performances are achieved by $\mathrm{DL}_{2}$, which is followed by $\mathrm{SL}_{2}$. On the other hand, $\mathrm{SL}_{1}$ and $\mathrm{DL}_{1}$ rank the last two. 

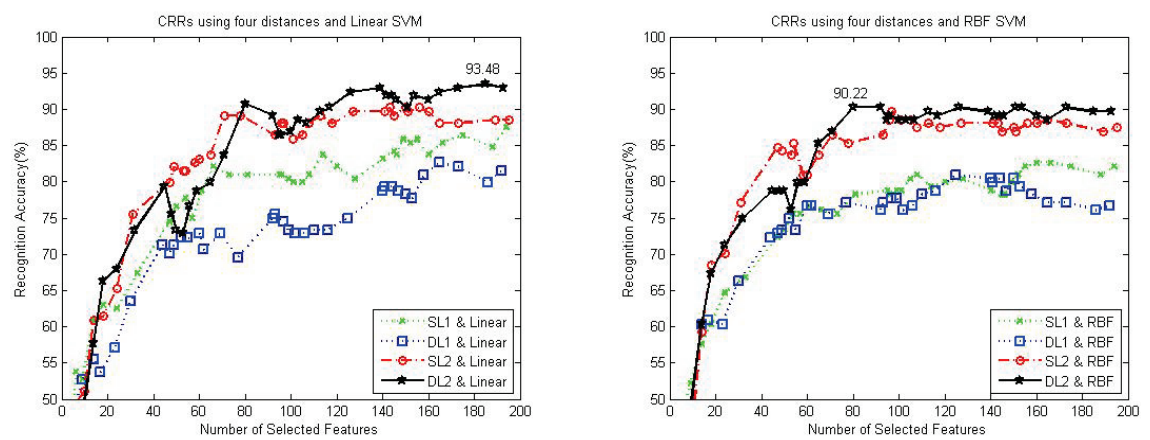

Fig. 4. Relationship between the CRR and the number of features on JAFFE.

\subsection{Performance on the CK Database}

Fig. 5 illustrates the relationship between the CRR and the number of features on the CK database. Seen from this figure, the proposed approach obtains the highest CRR of $94.48 \%$ using $\mathrm{DL}_{2}$ and RBF SVM when the error threshold is 0 and the number of features is 180 . This may imply that a performance improvement can be achieved once using a larger number of features. The relationship is similar to that of JAFFE in that the CRR grows up rapidly at starting stage and $\mathrm{L}_{2}$ distances outperform $\mathrm{L}_{1}$ distances for both linear and RBF. On the other hand, the CRR reaches a plateau with a speed quicker than that of JAFFE and $\mathrm{DL}_{1}$ performs better than $\mathrm{SL}_{1}$. Moreover, the performance difference between linear and RBF is smaller than that of JAFFE.
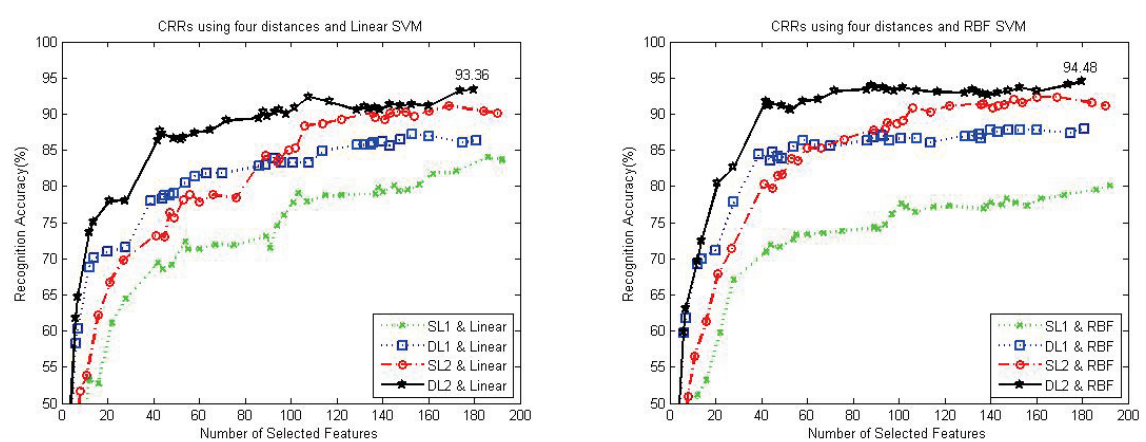

Fig. 5. Relationship between the CRR and the number of features on CK.

\subsection{Performance with and without Matching Area}

To evaluate the performance improvement rising from the use of the dynamic features, we compare the performances obtained with and without matching area. Note that the features obtained without matching area are supposed to not include dynamic 
information of position, scale and shape changes. Fig. 6 shows the comparison results when the error threshold equals to 0 . The results of JAFFE (left) and CK (right) are obtained using linear and RBF SVMs respectively. As can be seen, for JAFFE, the performances of 4 distance metrics are greatly boosted due to the use of matching area. There is a CRR increase of $11.41 \%$ using $\mathrm{DL}_{2}$. For CK, the CRRs of $\mathrm{DL}_{1}$ and $\mathrm{DL}_{2}$ are improved about $2.5 \%$ due to the use of matching area, while the CRRs of $\mathrm{SL}_{1}$ and $\mathrm{SL}_{2}$ does not benefit from using matching area. Considering the highest CRR of 4 metrics, we can see that dynamic features help to improve the performance.
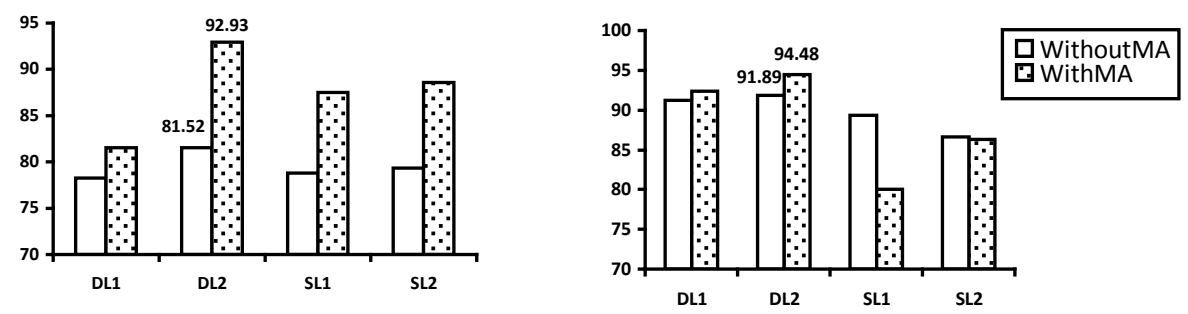

Fig. 6. Recognition accuracy (\%) obtained with and without matching area (MA).

\subsection{Comparison with State-of-the-Art Performance}

To evaluate the effectiveness of using dynamic, subtle and regional features, we also compare with previous approaches, which produce the state-of-the-art performances using the same databases and similar test strategies to our approach. As shown in Table 2, the proposed approach outperforms all 7 benchmarked approaches on JAFFE, and 2 out of the 4 benchmarked approaches on CK. The result using CK is $0.62 \%$ lower than the result obtained in [2]. However, the approach in [2] normalizes facial images based on manually-labeled eye locations, while the proposed approach is only based on rough face location. The result using CK is $1.39 \%$ lower than the result in [8]. But the approach in [8] obtains the result based on 5 -fold cross validation and 5 emotions, therefore, it uses more training images to classify less emotions compared to our approach.

Table 2. Comparison with state-of-the-art performance.

\begin{tabular}{ccccc}
\hline & Emotion Number & Feature & JAFFE & CK \\
\hline Proposed & 6 & patch-based Gabor & $\mathbf{9 3 . 4 8 \%}$ & $\mathbf{9 4 . 4 8 \%}$ \\
{$[9], 2005$} & 6 & fuzzy integral & $83.2 \%$ & - \\
{$[10], 2006$} & 6 & KCCA & $77.05 \%$ & - \\
{$[11], 2008$} & 7 & WMMC & $65.77 \%$ & - \\
{$[12], 2009$} & 7 & SFRCS & $85.92 \%$ & - \\
{$[13], 2005$} & 7 & Gabor + FSLP & $91.0 \%$ & - \\
{$[8], 2008$} & 7 (JAFFE), 5(CK) & FEETS + PRNN & $83.84 \%$ & $95.87 \%$ \\
{$[2], 2009$} & 7 (JAFFE), 6(CK) & boosted-LBP & $81.0 \%$ & $95.1 \%$ \\
{$[1], 2006$} & 7 & Gabor & - & $93.3 \%$ \\
{$[14], 2008$} & 7 & Gabor + Haar & - & $93.1 \%$ \\
\hline
\end{tabular}




\section{Conclusion}

The paper proposes a novel FER approach to improve the recognition performance using dynamic, subtle and regional features, which are obtained based on patch-based Gabor features and patch matching operations. The experimental results demonstrate good performances of the proposed approach on both the JAFFE and CK databases, and show big performance improvements due to the use of dynamic features. In addition, the comparison with previous approaches confirms the state-of-the-art performance using the proposed dynamic, subtle and regional features. The future work includes adopting real face detectors and testing on seven facial expressions.

\section{References}

1. Littlewort, G., Bartlett, M.S., Fasel, I., Susskind, J., Movellan, J.: Dynamics of facial expression extracted automatically from video. Image and Vision Computing 24 (2006) 615625

2. Shan, C., Gong, S., McOwan, P.W.: Facial expression recognition based on Local Binary Patterns: A comprehensive study. Image and Vision Computing 27 (2009) 803-816

3. Yang, P., Liu, Q., Metaxas, D.N.: Boosting encoded dynamic features for facial expression recognition. Pattern Recognition Letters 30 (2009) 132-139

4. Xiang, T., Leung, M.K.H., Cho, S.Y.: Expression recognition using fuzzy spatio-temporal modeling. Pattern Recognition 41 (2008) 204-216

5. Serre, T., Wolf, L., Bileschi, S., Riesenhuber, M., Poggio, T.: Robust Object Recognition with Cortex-Like Mechanisms. Pattern Analysis and Machine Intelligence, IEEE Transactions on 29 (2007) 411-426

6. Lyons, M., Akamatsu, S., Kamachi, M., Gyoba, J.: Coding facial expressions with Gabor wavelets. Automatic Face and Gesture Recognition, 1998. Proceedings. Third IEEE International Conference on (1998) 200-205

7. Kanade, T., Cohn, J.F., Yingli, T.: Comprehensive database for facial expression analysis. Automatic Face and Gesture Recognition, 2000. Proceedings. Fourth IEEE International Conference on (2000) 46-53

8. Wong, J.-J., Cho, S.-Y.: A face emotion tree structure representation with probabilistic recursive neural network modeling. Neural Computing \& Applications (2008)

9. Yuwen, W., Hong, L., Hongbin, Z.: Modeling facial expression space for recognition. Intelligent Robots and Systems, 2005. (IROS 2005). 2005 IEEE/RSJ International Conference on (2005) 1968-1973

10.Wenming, Z., Xiaoyan, Z., Cairong, Z., Li, Z.: Facial expression recognition using kernel canonical correlation analysis (KCCA). Neural Networks, IEEE Transactions on 17 (2006) 233-238

11.Zhengdong, C., Bin, S., Xiang, F., Yu-Jin, Z.: Automatic coefficient selection in Weighted Maximum Margin Criterion. Pattern Recognition, 2008. ICPR 2008. 19th International Conference on (2008) 1-4

12.Kyperountas, M., Tefas, A., Pitas, I.: Salient feature and reliable classifier selection for facial expression classification. Pattern Recognition 43 972-986

13.Guo, G., Dyer, C.R.: Learning from examples in the small sample case: face expression recognition. IEEE Transactions on Systems, Man, and Cybernetics, Part B 35 (2005) 477488

14.Chen, H.Y., Huang, C.L., Fu, C.M.: Hybrid-boost learning for multi-pose face detection and facial expression recognition. Pattern Recognition 41 (2008) 1173-1185 